\title{
论 文
}

\section{内蒙古二连地区古近纪早期地层序列、哺乳动物 演化及其环境响应}

\author{
王元青 ${ }^{(1)}$ ，孟津 ${ }^{(2)}$, Christopher K. BEARD ${ }^{(3)}$ ，李茜 ${ }^{(1)}$ ，倪喜军 ${ }^{(1)}$, Daniel L. GEBO ${ }^{(4)}$, \\ 白滨 ${ }^{1}$, 金迅 $^{(1)}$, 李萍 ${ }^{11}$
}

(1) 中国科学院古脊椎动物与古人类研究所, 脊椎动物进化系统学重点实验室, 北京 100044 ;

(2) American Museum of Natural History, New York 10024, USA;

(3) Carnegie Museum of Natural History, Pittsburgh 15213, USA;

(4) Northern Illinois University, DeKalb, 60115, USA

*E-mail: wangyuanqing@ivpp.ac.cn

收稿日期: 2010-06-11; 接受日期: 2010-09-02

国家重点基础研究发展计划项目(编号：2006CB806400)、国家自然科学基金重点项目(批准号：40532010)、科技部基础性专项(编号： 2006FY120300-15)和中国科学院化石发掘与修理专项基金以及美国科学基金项目(编号: EAR-0120727, BCS-0309800)资助

\begin{abstract}
摘要 内蒙古二连盆地呼和勃尔和地区古近系包括 3 个组: 脑木根组、阿山头组和伊尔丁曼 哈组, 从中可以划分出 12 个含哺乳动物化石的层位, 其中脑木根组 4 个, 阿山头组 6 个, 伊尔 丁曼哈组 2 个. 研究表明, 美国中亚考察团在这一地区划分的“呼尔井组”实际上是伊尔丁曼哈 组, 而“伊尔丁曼哈组”则为阿山头组. 根据最新的古地磁资料, 对相关组的时代和哺乳动物分 期的延续时间进行了重新厘定, 指出脑木根组上部包含了早始新世地层, 阿山头组绝大部分属 于早始新世，而不是过去认为的中始新世. 将格沙头期、伯姆巴期和阿山头期分别与国际地质 年表的坦尼特期、伊普里斯期早期和伊普里斯期中晚期至鲁帝特期最早期相对比，同时认为它 们分别与北美古近纪提法尼期晚期至克拉克福克期、华沙溪期早期和华沙溪期中晚期及勃里吉 期大部相当. 古近纪早期, 哺乳动物群演替以新科出现和属种更替为主, 与这一时期大多数情 况下气候的逐渐变化相对应; 而众多目一级现代哺乳动物类群在始新世之初的突然出现则很 可能与发生在古新世-始新世之交的高温事件有关.
\end{abstract}

关键词

二连盆地

古近纪

哺乳动物

演化

生物年代学

环境响应
蒙古高原是古近纪古脊椎动物学和地层学研究 的重要地区之一. 自 20 世纪 20 年代美国自然历史博 物馆中亚考察团(以下简称中亚考察团)在这一地区 开展考察以来, 发现了为数众多的哺乳动物化石地 点和大量化石标本 ${ }^{[1]}$. 大多数亚洲古近纪哺乳动物分 期都是根据这一地区发现的哺乳动物群提出的 ${ }^{[1,2]}$.
这些哺乳动物分期构成了亚洲哺乳动物年代学的框 架, 成为东亚地区古近纪相关研究的标尺, 被广泛运 用于区域及洲际陆相古近系对比以及相关问题(如古 近纪哺乳动物群演替和古动物地理学等) 的讨论 中 $^{[3 \sim 7]}$. 然而, 由于缺乏对亚洲古近纪陆相哺乳动物 分期的年代界定，从而不仅影响了洲际生物地层对

引用格式: Wang Y Q, Meng J, Beard C K, et al. Early Paleogene stratigraphic sequences, mammalian evolution and its response to environmental changes in Erlian Basin, Nei Mongol, China. Sci China Earth Sci, 2010, doi: 10.1007/s11430-010-4095-8 
比, 而且妨碍了对主要哺乳动物类群演化历史的认 识.

中国内蒙古中部地区, 特别是二连盆地, 古近纪 地层相当发育 ${ }^{[8]}$, 哺乳动物化石也极为丰富 ${ }^{[1,9]}$, 是许 多古近纪地层单位的命名地点, 亚洲陆相哺乳动物 分期中有不少是建立在这一地区哺乳动物群的基础 之上的 ${ }^{[2,7,10,11]}$, 因此二连盆地在亚洲古近纪哺乳动 物生物地层学、年代学研究中有着举足轻重的地位. 由于陆相地层横向变化较大, 而且几乎所有命名地 点的地层出露都不完整, 以往的工作中有关岩石地 层划分对比存在诸多错误, 进而造成了动物群组成 的混淆. 虽然过去已经认识到这一问题的存在, 并做 了一定的努力, 但并未真正解决这一问题 ${ }^{[12 ~ 15] . ~}$

近年来, 中国科学院古脊椎动物与古人类研究 所考察队在内蒙古二连盆地东部进行了大量的野外 工作, 澄清了一系列长期存在的岩石地层学和生物 地层学方面的问题 ${ }^{[16]}$, 完善了二连盆地东部生物地 层序列, 并结合磁性地层学研究 ${ }^{[17]}$, 建立了哺乳动物 年代框架.

\section{1 地质背景}

二连盆地位于内蒙古自治区中部, 紧邻中蒙边 界(图 1), 盆地内古近纪地层发育. 在其东部出露的 主要为古近系下部, 包括 3 个组: 脑木根组、阿山头 组和伊尔丁曼哈组 ${ }^{[14,16,18]}$.

脑木根组的命名地点位于四子王旗脑木更苏木 的海流图(哈留特), 为砂岩、泥质砂岩和砂质泥岩为 主的地层 ${ }^{[18,19]}$. 阿山头组和伊尔丁曼哈组的命名地 点都在二连浩特市东南约 $30 \mathrm{~km}$ 的伊尔丁曼哈陡坎. 剖面上出露的地层最初全部被归入伊尔丁曼哈组 ${ }^{[20]}$. 1924 年, 剖面下部的红色泥岩和粉砂岩被单独划分 出来, 并依 $11 \mathrm{~km}$ 以外的阿山头敖包将其暂时称为阿 山头组 ${ }^{[21]}$. 后来, 明确将下部的红色泥岩和粉砂岩称 为阿山头组 ${ }^{[22]}$. 这样, 伊尔丁曼哈组就只限于剖面上 部的白色砂质泥岩、砂岩和砾岩.

脑木根组的时代过去一直认为是晚古新 $世^{[10,19,23,24]}$. 在巴彦乌兰地区，曾报道巴彦乌兰组的 时代为早始新世, 但所列的哺乳动物化石则都是古

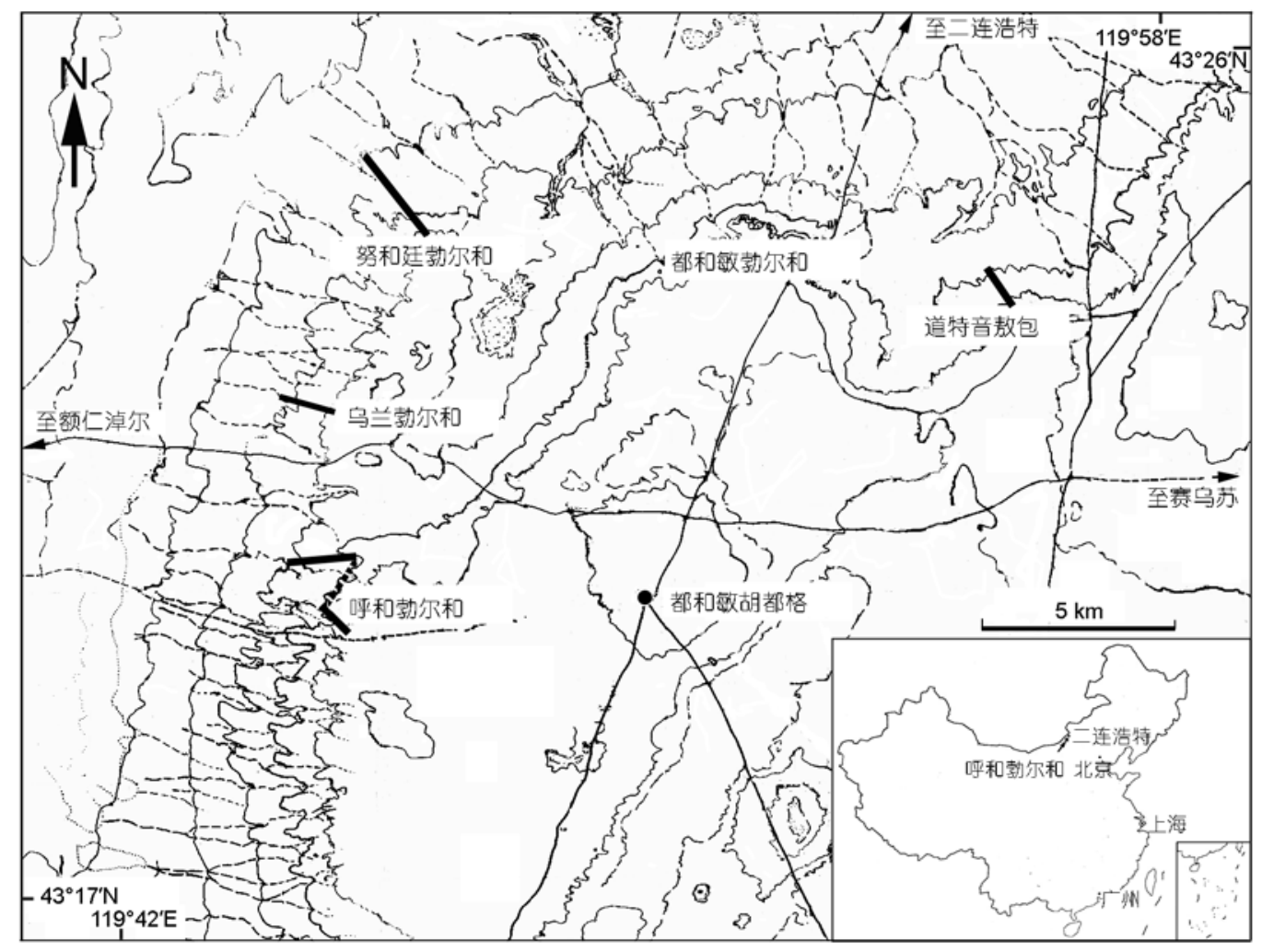

图 1 内蒙古二连盆地呼和勃尔和地区及剖面位置图

据文献[16]修改 
新世的典型属种 ${ }^{[18]}$. 后来的研究认为, 巴彦乌兰组很 可能是脑木根组的相变, 时代应该是古新世 ${ }^{[25]}$. 阿山 头组的时代被认为是中始新世 ${ }^{[10]}$ 或早-中始新世 ${ }^{[7,11]}$, 伊尔丁曼哈组则属于中始新世 ${ }^{[7,9 \sim 11]}$. 直到在乌兰勃 尔和脑木根组上部发现 Gomphos elkema等蒙古早始 新世典型化石之后, 才确定了二连盆地早始新世早 期地层的存在 ${ }^{[16]}$.

在现称的呼和勃尔和地区 (大致相当于中亚考察 团马捷茨营地及周边地区), 我们实测了 4 条剖面, 分 别位于道特音敖包、努和廷勃尔和、乌兰勃尔和以及 呼和勃尔和(图 1). 根据努和廷勃尔和与呼和勃尔和 两条实测剖面, 可以综合成一条完整剖面, 代表了这 一地区出露的全部古近纪地层(图 2). 综合剖面的厚 度为 $82.5 \mathrm{~m}$, 两个假整合面将其划分为 3 个部分. 根 据层位关系、与命名剖面的岩性对比, 并结合哺乳动 物化石, 认为自下而上依次是: 脑木根组、阿山头组 和伊尔丁曼哈组 ${ }^{[16]}$, 厚度分别是 $37.5,35.2$ 和 $9.8 \mathrm{~m}$. 综合剖面的岩性描述如下:

\section{伊尔丁曼哈组}

18）基部为灰白色及黄绿色细砂岩夹泥砾, 向 上是砂质砾岩夹少量泥岩, 并含有分选、磨圆较差的 黑色岩屑. 化石丰富, 包括 Lophialetes expeditus 和 Protitan sp. 在平台顶部或戈壁滩等相同层位, 是砂 岩和分选与磨圆均较好的石英组成的砾岩层. $4.2 \mathrm{~m}$

17) 灰绿色泥质砂岩和粗砂岩, 包含白色结核 和来自下伏地层的红褐色泥岩团块; 薄层黄绿色砂 质泥岩和砂岩, 夹有砾岩和红色砂质泥岩透镜体. 与 下伏地层接触面极不平整. 化石丰富, 包括 Tarkops mckennai, Propterodon morrisi, Harpagolestes leei, Andrewsarchus mongoliensis, Lophialetes expeditus, Deperetella sp., Gomphos shevyrevae, Tamquammys sp., Asiomys sp., Cricetidae, Yuomyidae 和 Lagomorpha.

$5.6 \mathrm{~m}$

------ 假整合(上部沉积间断)

阿山头组

16) 杂色(黄绿色及红棕色) 泥质砂岩夹粉砂岩 及砂质泥岩. 产 Schlosseria magister.

$4.9 \mathrm{~m}$

15) 灰绿色及杂色粉砂岩和细砂岩, 层理发育, 底部与下伏地层接触面局部不平整. 含有来自下伏 地层的泥岩团块和奇蹄类的化石. 产 Schlosseria magister, Gobiatherium mirificum, Fostercooperia confluens, Tamquammys sp.和 Yuomyidae.

14）浅红棕色泥岩或粉砂岩, 表面有黑色锈斑. 一些层位砂质较多, 最上部约 $30 \mathrm{~cm}$ 为红褐色泥岩. 产Schlosseria magister 及 Teleolophus sp. $2.1 \mathrm{~m}$

13）浅肉红色或红棕色泥质粉砂岩夹砂岩透镜 体及少量泥岩, 层理发育, 化石丰富. 产 Schlosseria magister, Tamquammys sp.和 Yuomyidae. $\quad 8.1 \mathrm{~m}$

12) 红色砂质泥岩夹少量粉砂岩, 有黑斑及锰 质结核. Schlosseria magister 非常丰富, 同时产有 Sinosinopa sinensis, Archetypomys erlianensis, Erlianomys combinatus, Tamquammys wilsoni, Advenimus burkei, Apternodontidae 和 Lagomorpha 等化石. $2.7 \mathrm{~m}$

11) 杂色粉砂岩, 夹有细砂岩和泥岩. 有骨头碎 片和硅化木, 多来自下伏的灰绿色地层. $\quad 4.4 \mathrm{~m}$

10）河流相砂岩、砾岩夹细岩屑和交错层理发育 的砂、泥岩, 砾石成分为泥质及长英质. 含 Archetypomys erlianensis, Erlianomys combinatus, Advenimus burkei, Tamquammys wilsoni, Paramyidae, Apternodontidae, Dawsonolagus antiquus, Gobiatherium mirificum, Metacoryphodon luminis, Litolophus gobiensis, Schlosseria magister, Teleolophus sp., Hyrachyus sp. 以及 Mesonyx üqbulakensis 化石.

$5.3 \mathrm{~m}$

------- 假整合(下部沉积间断) -------

脑木根组

9) 红棕色及黄绿色砂质泥岩, 底部含有灰白色 钙质结核, 上部地层多为杂色(红褐色及灰绿色)泥岩 且含较多砂质成分, 表面风化呈棕褐色. 产有 Gomphos elkema, Baataromomys ulaanus, Anatolostylops zhaii, Pataecops parvus, Lophialetidae gen. et sp. nov., Ctenodactyloidea gen. et sp. nov.及 Lagomorpha等化石. 该层底部往上约 $5 \mathrm{~m}$ 处还产有Uintatherium sp.

$7 \mathrm{~m}$

8) 下部肉红色及黄绿色泥质砂岩, 上部以红棕 色泥岩为主, 含有黑色锈斑及锰质结核. $6.2 \mathrm{~m}$

7) 含有 $0.5 \sim 1 \mathrm{~mm}$ 白色小结核的肉红色及土褐 色疏松泥质粉砂岩或粉砂质泥岩; 向上岩性为呈牛 肉色的致密胶结泥岩, 包含钙质和砂质团块. 在小山 包顶部有一厚度 5 10 cm、含有白色结核的不连续透 镜体. 产 Lambdopsalis bulla 及 Pastoralodon lacustris 等化石.

$8.6 \mathrm{~m}$

6) 红棕色及黄绿色泥质砂岩及粗砂岩. $2.7 \mathrm{~m}$ 
5) 下部为层理发育的肉红色砂质泥岩, 含有粒 径 2 20 mm, 以小颗粒为主的结核. 向上过渡为细粒 含砾砂岩夹泥岩.

$2.8 \mathrm{~m}$

4) 灰白色和绿色细砂岩, 局部含粉砂岩及泥岩, 水平层理发育. 下部地层含有粒径范围 5 40 $\mathrm{mm}$ 的 白色钙质结核, 许多部位含有大量硅化木碎片. 地层 向上过渡为杂色 (棕灰色、绿色) 粉砂质泥岩, 层理清 晰.

$1.7 \mathrm{~m}$

3) 杂色 (土黄色、浅红色) 砂质泥岩, 含有结构 疏松的泥岩团块. 可见硅化木碎片, 哺乳动物化石丰 富, 有 Lambdopsalis bulla, Prionessus lucifer, Bayanulanius tenuis, Tribosphenomys minutus, Eomylus borealis, Prodinoceras xinjiangensis 及 Palaeostylops iturus 等.

$3.4 \mathrm{~m}$

2) 浅红棕色疏松泥质砂岩, 含有小结核, 层理 不发育, 夹有黄绿色砂岩透镜体和细层理发育的粉 砂岩, 风化表面呈咖啡色.

$2.9 \mathrm{~m}$
1) 浅灰绿色疏松砂质粘土, 含有细岩屑, 上覆 有 $5 \sim 10 \mathrm{~cm}$ 厚的坚硬砂岩层.

$2.2 \mathrm{~m}$

下伏地层掩盖

总体来说，脑木根组以红色泥岩、粉砂质泥岩以 及砂岩夹层为主. 阿山头组底部为含砾粗砂岩, 往上 主要是泥质粉砂岩. 伊尔丁曼哈组主要由含砾粗砂 岩构成. 经过在呼和勃尔和地区的详细工作, 并对比 中亚考察团的野外记录, 可以肯定中亚考察团的马 捷茨营地附近重要化石地点的位置. 马捷茨营地位 于现称为都和敏勃尔和的陡坎边缘的某个地点; 马 捷茨营地以西 $9.6 \mathrm{~km}$ (6 miles west of Camp Margetts) 地点应该是现在的努和廷勃尔和; 马捷茨营地西南 $16 \mathrm{~km}$ (10 miles west of Camp Margetts)地点应该是呼 和勃尔和; 马捷茨营地西南 $235^{\circ} 11 \mathrm{~km}$ (7 miles at $235^{\circ}$ of Camp Margetts)和西 $11 \mathrm{~km}$ (7 miles west of Camp Margetts)分别是乌兰勃尔和和一个被暂时称为 “乌兰勃尔和北”的地点. 与相关组命名剖面的岩性

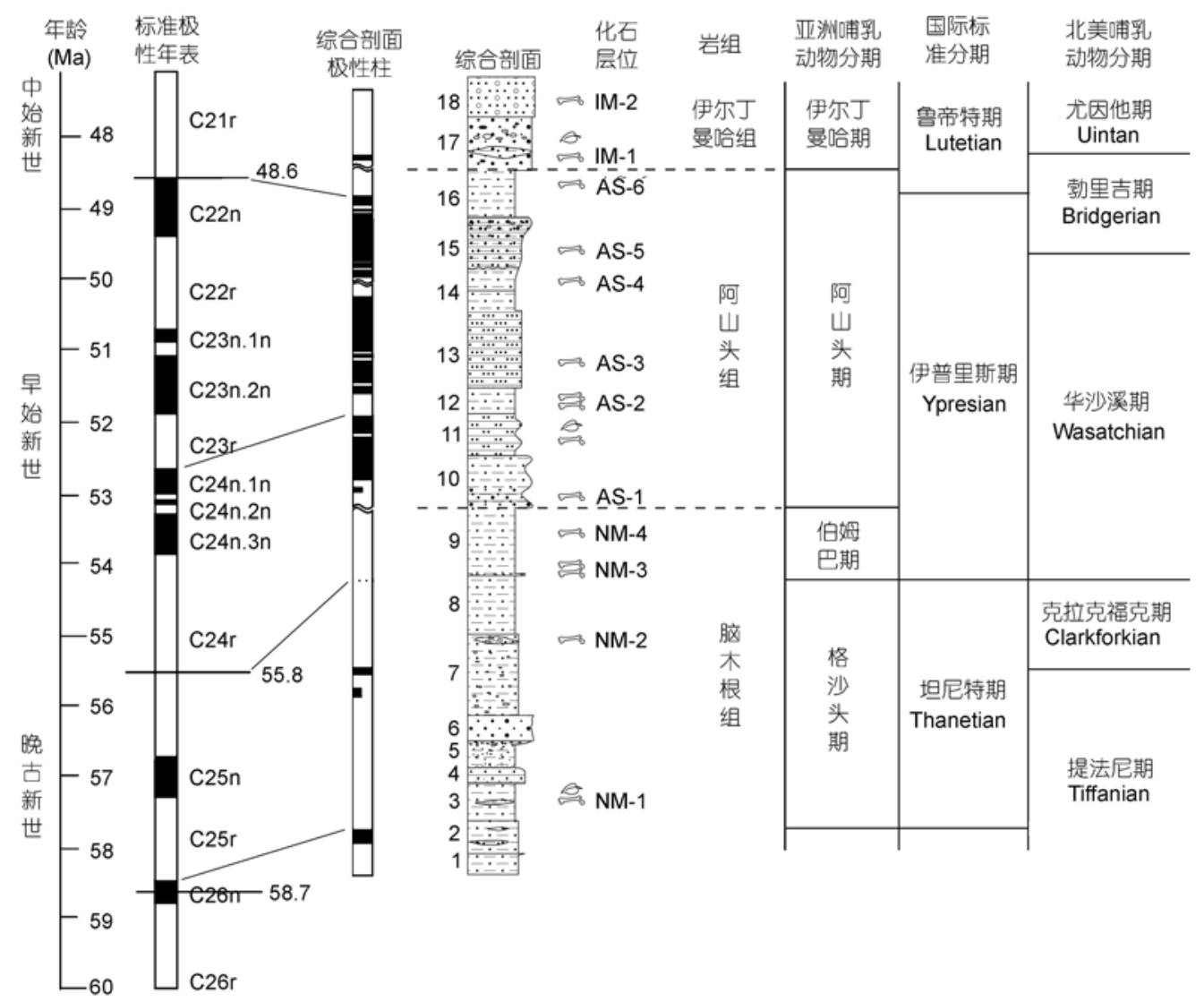

图 2 二连盆地东部古近纪地层、磁性柱、哺乳动物层位及其国际对比 古地磁资料引自文献[17], 地层剖面修改自文献[16] 
对比 (结合生物化石), 显示中亚考察团在马捷茨营地 及周边地区的所谓“呼尔井组”实际上是伊尔丁曼哈 组, 而 “伊尔丁曼哈组”则为阿山头组. 这些看法与前 人的基本一致, 但需要说明的是, 以前的文献中对于 有关地点和层位存在误读. 马捷茨营地曾被认为位 于现今的呼和勃尔和一带, 而其西南及以西的各个 地点则被认为位于巴彦乌兰一带 ${ }^{[14]}$, 后来的研究者 指出了这一错误, 并认为马捷茨营地位于都和敏勃 尔和陡坎边缘 ${ }^{[15]}$. 在最近的工作中, 乌兰勃尔和也曾 被误以为是呼和勃尔和, 剖面顶部灰白色含砾中粗 粒砂岩也被误认为是伊尔丁曼哈组 ${ }^{[26,27]}$. 后来, 因将 1930 年Granger野外记录中马捷茨营地以西 $11 \mathrm{~km}$ 地 点的剖面图误读为西南 $235^{\circ} 11 \mathrm{~km}$ 者, 从而认为美国 中亚考察团马捷茨营地及附近地区 (即呼和勃尔和地 区) 的 “伊尔丁曼哈组, 属于阿山头组和/或脑木根 组” ${ }^{[16]}$. 实际上, 通过分析对比我们最近野外工作中 所获得的资料与美国中亚考察团的野外记录 ${ }^{[28]}$, 可 以认为这一地区中亚考察团原认为属于“伊尔丁曼哈 组”的化石中并没有来自脑木根组的化石.

\section{2 哺乳动物化石产出层位}

二连盆地下古近系的一些层位中哺乳动物化石 比较丰富. 过去, 标本采集都是以组为单位进行的, 同一个组中产出的化石都被归入到一个动物群

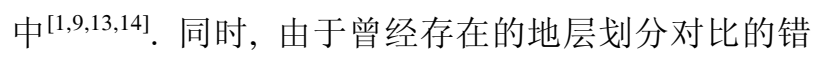
误, 相关动物群的组成上出现了混乱的状况. 在野外 工作中, 我们在仔细划分地层的基础上, 系统地分层 采集了哺乳动物化石, 在呼和勃尔和地区共划分出 12 个含哺乳动物化石的层位, 其中脑木根组 4 个, 阿 山头组 6 个, 伊尔丁曼哈组 2 个. 按照产出层位, 自 下而上分别编号为NM-1 至NM-4, AS-1 至AS-6 以及 IM-1 和IM-2 (图 2). 各个层位已经发表或鉴定出的哺 乳动物化石有:

IM-2: 综合剖面第 18 层, 产奇蹄类 Lophialetes expeditus, Protitan sp.

IM-1：综合剖面第 17 层, 产灵长类Tarkops mckennai ${ }^{[29]}$, 肉齿类 Propterodon morrisi, 中兽类 Harpagolestes leei, Andrewsarchus mongoliensis, 奇蹄 类Lophialetes expeditus, Deperetella sp., 啮形类 Gomphos shevyrevae ${ }^{[30]}$, 啮齿类 Tamquammys sp., Asiomys sp., Cricetidae, Yuomyidae, 兔形类.
AS-6: 综合剖面第 16 层, 产奇蹄类 Schlosseria magister.

AS-5: 综合剖面第 15 层, 产奇蹄类 Schlosseria magister, Fostercooperia confluens, 恐角类 Gobiatherium mirificum, 啮齿类 Tamquammys sp., Yuomyidae.

AS-4: 综合剖面第 14 层, 产奇蹄类 Schlosseria magister, Teleolophus sp.

AS-3: 综合剖面第 13 层, 产奇蹄类 Schlosseria magister, 啮齿类 Tamquammys sp., Yuomyidae.

AS-2: 综合剖面第 12 层, 产奇蹄类Schlosseria magister $^{[31]}$, 兔形类Dawsonolagus antiquus, 啮齿类 Archetypomys erlianensis ${ }^{[32]}$, Erlianomys combinatus ${ }^{[33]}$, Tamquammys wilsoni, Advenimus burkei, 食虫类 Sinosinopa sinensis, Apternodontidae.

AS-1：综合剖面第 10 层, 产啮齿类Archetypomys erlianensis ${ }^{[32]}$, Erlianomys combinatus ${ }^{[33]}$, Tamquammys wilsoni, Advenimus burkei, Paramyidae, 兔形类Dawsonolagus antiquus ${ }^{[34]}$, 食虫类Apternodontidae, 奇蹄类Litolophus gobiensis ${ }^{[35]}$, Schlosseria magister, Teleolophus sp., Hyrachyus sp., 恐角类 Gobiatherium mirificum ${ }^{[36]}$, 全齿类Metacoryphodon luminis, 中兽类Mesonyx üqbulakensis.

NM-4: 综合剖面第 9 层上部, 产啮形类Gomphos elkema, 恐角类Uintatherium sp. ${ }^{[36]}$, 奇蹄类Pataecops parvus, 啮齿类Ctenodactyloidea indet.

NM-3：综合剖面第 9 层底部, 产啮形类Gomphos elkema $^{[27]}$, 灵长类Baataromomys ulaanus ${ }^{[37]}$, 北柱兽类 Anatolostylops zhail ${ }^{[38]}$, 中兽类Dissacus sp., 奇蹄类 Pataecops parvus, 脊齿貘科一新种 ${ }^{[39]}$, 梳趾鼠类一 新种, 一种待定兔形目.

NM-2: 综合剖面第 7 层顶部, 产全齿类 Pastoralodon lacustris, 多瘤齿兽类 Lambdopsalis bulla.

NM-1：综合剖面第 3 层, 产多瘤齿兽类 Lambdopsalis bulla, Prionessus lucifer, 北柱兽类 Palaeostylops iturus, 恐角兽类 Prodinoceras xinjiangensis, 食虫类 Bayanulanius tenuis, 假古猬类 Pseudictops lophiodon, 啮形类 Tribosphenomys minutes.

除了前面列出的哺乳动物化石之外, 美国中亚 考察团发现于呼和勃尔和地区并认为产于“伊尔丁曼 哈组”的化石应该产于阿山头组. 层位应该属于阿山 头组的化石还包括: 中兽类 Mongolonyx dolichogna- 
thus ${ }^{[40]}$, 肉齿类Sarkastodon mongoliensis ${ }^{[41]}$, 奇蹄类 Metatelmatheirum cristatum $^{[22]}$ 和Helaletes fissus ${ }^{[43]}{ }^{\text {等. }}$ 而原认为产于“呼尔井组”的化石则多数产于伊尔丁 曼哈组.

此外, 在巴彦乌兰地区以及苏崩地区发现的脑 木根组化石 ${ }^{[25,44 \sim 47]}$, 其层位应该与NM-1 大体相当.

根据现有的哺乳动物化石资料, 内蒙古二连盆 地呼和勃尔和地区的古新统与始新统界线应该位于 NM-2 和NM-3 两个化石层之间 ${ }^{[48]}$. 但由于没能获得 稳定碳同位素的证据, 无法准确确定古新统和始新 统界线. 衡东岭茶地区岭茶组所产的哺乳动物化石 中有不少与蒙古奈玛盖特盆地(Nemegt Basin)伯姆巴 期(Bumbanian)化石可以对比，被认为时代相当，而 NM-3 化石层亦与伯姆巴期化石层相当. 因此可以将 二连盆地含 Gomphos elkema等化石的层位与岭茶组 化石层间接对比. 而且岭茶组含哺乳动物化石的层 位中也富含钙质结核, 与含 Gomphos elkema等化石 的地层很相似 ${ }^{[48]}$. 古地磁和碳同位素研究显示, 岭茶 组哺乳动物化石层位于磁极性 $\mathrm{C} 24 \mathrm{r}$ 和 $\delta^{13} \mathrm{C}$ 负漂移的 时间段内, 因而可以认为其时代为早始新世最早 期 $^{[3,49]}$. 结合呼和勃尔和地区古近系的实际情况, 将 古新统和始新统界线置于NM-3 化石层之底(即综合 剖面第 9 层之底)应该是可以接受的.

\section{3 哺乳动物生物年代学}

新生代哺乳动物年代学研究以北美地区最有影 响, 自从Wood等 ${ }^{[50]}$ 提出以哺乳动物化石为基础的生 物地层框架——陆相哺乳动物分期(Land Mammal Ages) 以来, 相关研究在理论和实践方面都取得了重 要进展 ${ }^{[51,52]}$, 通过开展包括哺乳动物化石及生物地 层、古地磁、年代测定以及稳定同位素在内的综合研 究, 完善了陆相哺乳动物分期系统, 使之能够与国际 标准进行很好的对比 ${ }^{[53 \sim 56]}$, 同时将北美陆相哺乳动 物分期与全球性事件联系起来 ${ }^{[57]}$. 亚洲陆相哺乳动 物分期自提出 ${ }^{[2]}$ 以来, 经过多年的发展 ${ }^{[1,9,58,59]}$, 形成 了比较完整的亚洲陆相哺乳动物分期系统 ${ }^{[7]}$, 并被广 泛采用 ${ }^{[4]}$. 内蒙古二连盆地呼和勃尔和地区的古近纪 涉及到四个亚洲陆相哺乳动物分期——格沙头期 (Gashatan)、伯姆巴期、阿山头期(Arshantan)和伊尔 丁曼哈期(Irdinmanhan).

格沙头期源于蒙古乌兰诺尔盆地 (Ulan-Nur
Basin)格沙头组(Gashato Formation)一段的格沙头动 物群, 而伯姆巴期则源于蒙古奈玛盖特盆地娜兰布 拉克组 (Naran-Bulak Formation) 伯姆巴段 (Bumban Member)的动物群 ${ }^{[2]}$.

NM-1 和NM-2 两个层位产出的哺乳动物化石都 是典型的晚古新世格沙头期的代表, 所有化石均仅 见于中国和蒙古的相当地层中 ${ }^{[9,23 \sim 25,44,60]}$. NM-3 和 NM-4 两个层位发现的Gomphos elkema 是蒙古早始新 世伯姆巴期的典型代表 ${ }^{[27,61]}$. NM-3 产出的灵长类 Baataromomys ulaanus 与北美早始新世华沙溪期 (Wasatchian (Wa-0))发现的“Teilhardina” brandti ${ }^{[62]}$ 非 常相似, 后者被认为与内蒙古化石属于同一个属 ${ }^{[37]}$. 根据现有的化石资料, 可以认为呼和勃尔和地区 NM-1 和NM-2 两个层位产出的化石属于格沙头期, NM-3 和NM-4 产出的化石属于伯姆巴期.

阿山头期和伊尔丁曼哈期是根据内蒙古二连盆 地东部阿山头组和伊尔丁曼哈组产出的哺乳动物群 命名的 ${ }^{[2]}$, 因此从阿山头组和伊尔丁曼哈组中发现的 化石应该被分别看作是阿山头期和伊尔丁曼哈期动 物群的典型成员(图 2).

中国乃至亚洲古近纪陆相哺乳动物分期虽然已 经沿用了近 30 年, 但各分期的延续时限, 长期没有 界定. 虽然以前有少量的古地磁工作, 但并没有对相 关哺乳动物分期进行年代界定 ${ }^{[3,26,63,64]}$. 直到最近几 年, 才在中国部分古近纪地点开展了这一方面的工 作, 对相关的分期进行了界定, 但仍主要集中于中国 南方古新世较早的时间段 ${ }^{[65,66]}$. 而古近纪其他哺乳 动物分期的延续时间则主要是依据与北美的洲际生 物地层对比来推测的 ${ }^{[4,7,10,11]}$. 由于这一地区缺乏可 以用来进行同位素测年的样品, 古地磁就成了目前 所能采用的唯一手段. 根据呼和勃尔和地区最新的 古地磁研究结果 ${ }^{[17]}$, 我们可以对相关的哺乳动物分 期的延续时间进行比较准确的推断.

根据在努和廷勃尔和和呼和勃尔和两条剖面的 古地磁研究结果, 呼和勃尔和地区的古近系记录了 5 个正极性段与 6 个负极性段, 可以与标准磁极性年表 的C21r-C26r 之间的极性带对应 ${ }^{[17]}$. 目前, 由于没有 合适的剖面，古新世浓山期和格沙头期的界线并没 有很好地确定. 根据在江西池江盆地和广东南雄盆 地的古地磁工作, 证实浓山期地层主要位于地磁极 性的 C26r 中 ${ }^{[65,66]}$. 而在呼和勃尔和地区的古地磁研 
究结果显示, 较低的含格沙头期哺乳动物化石的地 层(NM-1)处于 $\mathrm{C} 25 \mathrm{r}$ 下部 ${ }^{[17]}$. 因此, 可以暂时将浓山 期和格沙头期的界线置于 $\mathrm{C} 26 \mathrm{n}$ 之底. 这样, 格沙头 期的延续时间为 58.8 55.8 Ma, 相当于磁极性年表的 $\mathrm{C} 26 \mathrm{n}-\mathrm{C} 24 \mathrm{r}$ 下部。伯姆巴期的延续时间大约为 55.8 54.8 Ma, 位于 $\mathrm{C} 24 \mathrm{r}$ 中. 阿山头期的延续时间为 $54.8 \sim 47.6 \mathrm{Ma}$, 相当于C24r-C21r. 伊尔丁曼哈组的顶 界并不清楚, 因此依据伊尔丁曼哈组所产的哺乳动 物化石而建立的伊尔丁曼哈期只能确定其底界位于 $\mathrm{C} 21 \mathrm{r}$ 上部 (图 2).

根据以上结果, 我们可以将相关的亚洲陆相哺 乳动物分期与国际地质年表和北美陆相哺乳动物分 期进行比较准确的对比. 与国际地质年表对比, 格沙 头期与坦尼特期大致相当, 伯姆巴期相当于伊普里 斯期早期, 阿山头期相当于伊普里斯期中晚期至鲁 帝特期最早期. 与北美陆相哺乳动物分期对比, 格沙 头期相当于提法尼期晚期至克拉克福克期, 伯姆巴 期相当于华沙溪期早期, 阿山头期与华沙溪期中晚 期及勃里吉期大部相当 (图 2).

\section{4 哺乳动物演化与环境响应}

海洋沉积的氧同位素记录显示, 在晚古新世晚 期至早始新世气候最适宜期(Early Eocene Climatic Optimum), 大洋温度处于逐渐上升的阶段, 期间发 生了古新世-始新世之交的以碳同位素负向漂移(CIE) 为标志的急速转折事件, 即古新世-始新世高温事件 (Paleocene-Eocene Thermal Maximum, 缩写为PETM). 早始新世气候最适宜期之后, 大洋温度逐渐下降, 直 至发生始新世/渐新世降温事件 ${ }^{[67]}$. 根据古地磁标定, 可以将与呼和勃尔和地区古近纪相关的陆相哺乳动 物分期与海洋氧碳同位素变化曲线进行间接的对比 (图 3).

根据哺乳动物化石在呼和勃尔和地区产出的层 位, 可以看出古近纪早期哺乳动物演化的一些基本 规律 (图 3). 在古新世格沙头期, 哺乳动物以古老类 群为主，如全齿类、恐角类、北柱兽类、多瘤齿兽类 等. 现代类群的祖先类型只占极少的一部分, 如啮齿 类的祖先类型—啮形类. 进入始新世伯姆巴期, 若

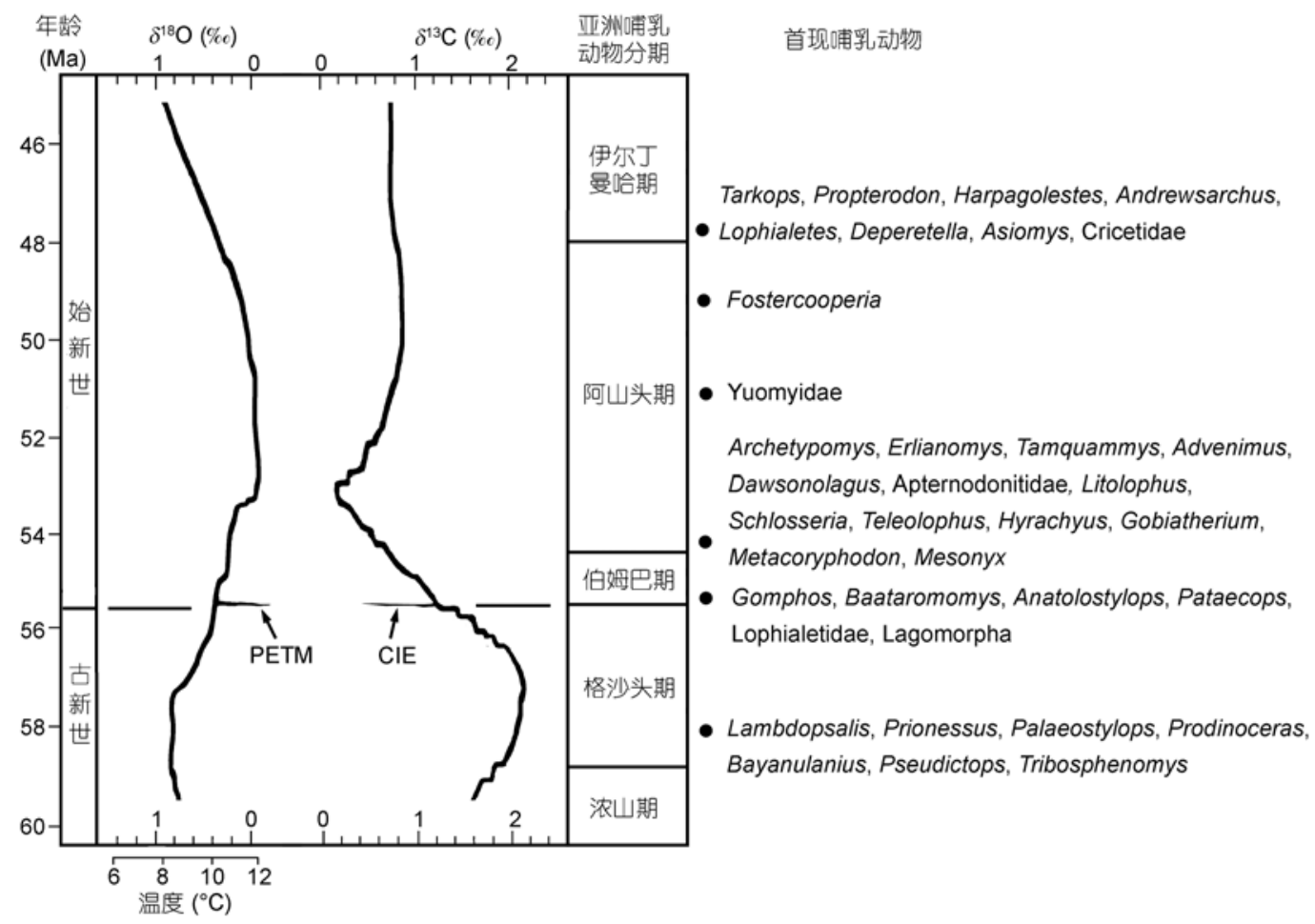

图 3 呼和勃尔和地区哺乳动物事件与全球环境变化的对应关系 氧碳同位素曲线依据文献[67]重绘, 哺乳动物科属在剖面中的首现位置以圆点(•)表示 
干现生的目一级哺乳动物类群出现, 其中包括奇蹄 目、真灵长类、啮齿目和兔形目等. 与此同时, 古老 类群也发生了属或科一级的变化(如全齿类、恐角类 和北柱兽类), 或区域性消失(如多瘤齿兽类), 但总体 来说, 在动物群中所占的比例和种数上都有明显的 下降 ${ }^{[11]}$. 值得指出的是伯姆巴期发现的跑犀科

Pataecops parvus 是迄今最早的犀超科代表, 同时还 有貘超科脊齿貘科的一个新种. 这两种化石都是世 界其他地区同时代地层中未曾发现过的种类. 考虑 到其他地区同时代地层中已经发现的奇蹄类爪兽超 科(蒙古)、马形类(北美) 以及等脊貘科(亚洲、北美、 欧洲)的代表这一事实, 可以认为, 奇蹄类在始新世 之初就开始了强烈的分异, 所有大的分支都已经出 现, 从而奠定了在整个始新世繁盛的基础. 在随后的 演化过程中, 古老类群进一步减少, 多数现生哺乳动 物类群表现出的是属种的更替, 尤其是奇蹄类和啮 齿类出现了众多新的科属(如奇蹄类的戴氏貘科、貘 犀科和雷兽科, 啮齿类的初鼠科、鼠齿类、豫鼠科和 仓鼠科), 成为始新世分异度最高的两个大类. 这样 的演化规律与其他地区的记录是吻合的 ${ }^{[7]}$.

哺乳动物在古近纪早期的这种演化历程, 在某 种程度上也反映了它们对环境变化的响应. 在晚古 新世至中始新世早期, 无论全球气温是升高还是下 降, 在绝大多数情况下都是一个渐变的过程, 因此哺 乳动物也表现出了逐渐发展和更替的演化历程. 然 而, 在古新世-始新世之交发生的高温事件却对哺乳 动物演化产生了重要的影响. 由于这次高温事件是 一个相对比较突然的事件, 全球平均气温短期内上 升了大约 $5^{\circ} \mathrm{C}^{[68]}$. 与此相对应的是, 陆生哺乳动物群
的组成发生了根本性的变化, 导致了众多目一级的 哺乳动物类群出现, 哺乳动物群也真正呈现出现代 面貌.

\section{5 结论}

(1) 纠正了地层划分方面的错误，澄清了部分哺 乳动物群组成方面的混乱. 认为原中亚考察团在马 捷茨营地及周边地区划分的“呼尔井组” 实际上是伊 尔丁曼哈组, 而“伊尔丁曼哈组”则为阿山头组. 与之 相关的阿山头动物群和伊尔丁曼哈动物群的组成也 应进行相应的厘定.

(2) 在呼和勃尔和地区古近纪地层中共划分出 12 个含哺乳动物化石的层位, 其中脑木根组 4 个 (NM-1 至 NM-4), 阿山头组 6 个(AS-1 至 AS-6), 伊尔 丁曼哈组 2 个(IM-1 和 IM-2).

(3) 根据最新的古地磁资料, 对相关组的时代和 哺乳动物分期的延续时间进行了重新厘定. 确认脑 木根组上部包含了早始新世地层, 阿山头组绝大部 分属于早始新世, 而不是过去认为的中始新世. 将格 沙头期、伯姆巴期和阿山头期分别与国际地质年表的 坦尼特期、伊普里斯期早期和伊普里斯期中晚期至鲁 帝特期最早期相对比，同时认为格沙头期、伯姆巴期 和阿山头期分别与北美陆相古近纪提法尼期晚期至 克拉克福克期、华沙溪期早期和华沙溪期中晚期及勃 里吉期大部相当.

(4) 呼和勃尔和地区的化石记录显示, 在古近纪 早期, 哺乳动物群演替以新科出现和属种更替为主, 发生在古新世-始新世之交的高温事件很可能是导致 现代面貌的哺乳动物群出现的主要因素.

致谢李传苝、周伟、曹强、谢树华、李士杰、李祁、李强、陈伟、高伟、李淳、王天宇参加野外工作; 童永生、 Mary R. Dawson 在部分化石的鉴定方面提供帮助, 李传蘩、童永生、邱占祥、王伴月与作者进行了有益的讨 论; 周伟、谢树华、李士杰、李祁、张丽芬、郭臣光、王阳花修理、篮洗和挑选化石, 审稿人提出修改建议, 在此一并表示衰心的感谢.

\section{参考文献}

1 Russell D E, Zhai R J. The Paleogene of Asia: Mammals and stratigraphy. Mém Mus Natl d'Hist Nat Sér C-Sci Terre, 1987, 52: 1-448

2 Romer A S. Vertebrate Paleontology. Chicago and London: University of Chicago Press, 1966. 1-467

3 Bowen G J, Clyde W C, Koch P L, et al. Mammalian dispersal at the Paleocene-Eocene boundary. Science, 2002, 295: 2062-2065

4 Luterbacher H P, Ali J R, Brinkhuis H, et al. The Paleogene Period. In: Gradstein F M, Ogg J G, Smith A, eds. A Geological Time Scale 2004. Cambridge: Cambridge University Press, 2004. 384-408 
5 Meng J, McKenna M C. Faunal turnovers of Palaeogene mammals from the Mongolian Plateau. Nature, 1998, 394: 364-367

6 Tsubamoto T, Takai M, Egi N. Quantitative analyses of biogeography and faunal evolution of Middle to Late Eocene mammals in East Asia. J Vert Paleont, 2004, 24: 657-667

7 Wang Y Q, Meng J, Ni X J, et al. Major events of Paleogene mammal radiation in China. Geol J, 2007, 42: 415-430

8 内蒙古自治区地质矿产局. 内蒙古自治区区域地质志. 中华人民共和国地质矿产部地质专报一一区域地质, 1991, 25: 1-725

9 Li C K, Ting S Y. The Paleogene mammals of China. Bull Carneg Mus Nat Hist, 1983, 21: 1-93

10 童永生, 郑绍华, 邱铸鼎. 中国新生代哺乳动物分期. 古脊椎动物学报, 1995, 33: 290-314

11 王元青, 孟津, 倪喜军, 等. 中国古近纪哺乳动物的辐射. 见: 戎嘉余, 等, 主编. 生物的起源、辐射与多样性演变一一华夏化石记录 的启示. 北京: 科学出版社, 2006. 735-755, 948-950

12 Radinsky L B. Notes on Eocene and Oligocene fossil localities in Inner Mongolia. Am Mus Novitat, 1964, (2180): 1-11

13 齐陶. 内蒙古呼和勃尔和陡坎晚始新世伊尔丁曼哈层及其哺乳动物群. 古脊椎动物学报, 1980, 18: 28-32

14 Qi T. The Middle Eocene Arshanto fauna (Mammalia) of Inner Mongolia. Ann Carnegie Mus, 1987, 56: 1-73

15 孟津. 双尖齿兽科(Didymoconidae)——新种及有关地点地层问题. 古脊椎动物学报, 1990, 28: 206-217

16 Meng J, Wang Y Q, Ni X J, et al. New stratigraphic data from the Erlian Basin: Implications for the division, correlation, and definition of Paleogene lithological units in Nei Mongol (Inner Mongolia). Am Mus Novitat, 2007, (3570): 1-31

17 孙勃, 岳乐平, 王元青, 等. 二连盆地下古近系的磁性地层学研究. 地层学杂志, 2009, 33: 62-68

18 江浩贤. 内蒙古二连盆地下第三系的划分. 内蒙古地质, 1983, 2: 18-36

19 周明镇, 齐陶, 李荣. 内蒙古四子王旗古新世地层及哺乳动物群的性质与对比. 古脊椎动物学报, 1976, 14: 228-233

20 Granger W, Berkey C P. Discovery of Cretaceous and older Tertiary strata in Mongolia. Am Mus Novitat, 1922, (42): 1-7

21 Berkey C P, Morris F K. Basin structures in Mongolia. Bull Amer Mus Nat Hist, 1924, 51: 103-127

22 Berkey C P, Morris F K. The Geology of Mongolia-A reconnaissance report based on the investigations of the years 1922-1923. In: Natural History of Central Asia. Vol. II. New York: American Museum of Natural History, 1927. 1-475

23 Wang Y Q, Hu Y M, Chow M C, et al. Chinese Paleocene mammal faunas and their correlation. In: Beard K C, Dawson M R, eds. Dawn of the Age of Mammals in Asia. Bull Carneg Mus Nat Hist, 1998, 34: 89-123

24 周明镇, 齐陶. 内蒙古四子王旗晚古新世哺乳类化石. 古脊椎动物学报, 1978, 12: 77-85

25 Meng J, Zhai R J, Wyss A R. The late Paleocene Bayan Ulan fauna of Inner Mongolia, China. In: Beard K C, Dawson M R, eds. Dawn of the Age of Mammals in Asia. Bull Carneg Mus Nat Hist, 1998, 34: 148-185

26 Bowen G J, Koch P L, Meng J, et al. Age and correlation of fossiliferous late Paleocene-early Eocene strata of the Erlian Basin, Inner Mongolia, China. Am Mus Novitat, 2005, (3474): 1-26

27 Meng J, Bowen G J, Ye J, et al. Gomphos elkema (Glires, Mammalia) from the Erlian Basin: Evidence for the early Tertiary Bumbanian Land Mammal Age in Nei-Mongol, China. Am Mus Novitat, 2004, (3245): 1-24

28 Granger W. Records of fossils collected in Mongolia. Central Asiatic Expeditions (Field Notes). New York: American Museum of Natural History, 1930. 1-163

29 Ni X J, Meng J, Beard K C, et al. A new tarkadectine primate from the Eocene of Inner Mongolia, China: Phylogenetic and biogeographic implications. Proc R Soc B-Biol Sci, 2010, 277: 247-256

30 Meng J, Kraatz B P, Wang Y Q, et al. A new species of Gomphos (Glires, Mammalia) from the Eocene of the Erlian Basin, Nei Mongol, China. Am Mus Novitat, 2009, 3670: 1-11

31 Li P, Wang Y Q. Newly discovered Schlosseria magister (Lophialetidae, Perissodactyla, Mammalia) skulls from central Nei Mongol, China. Vert PalAsiat, 2010, 48: 119-132

32 Meng J, Li C K, Ni X J, et al. A new Eocene rodent from the lower Arshto Formation in the Nuhetingboerhe (Camp Margetts) Area, Inner Mongolia. Am Mus Novitat, 2007, (3569): 1-18

33 Li Q, Meng J. Erlianomys combinatus, a primitive myodont rodent from the Eocene Arshanto Formation, Nuhetingboerhe, Nei Mongol, China. Vert PalAsiat, 2010, 48: 133-144

34 Li C K, Meng J, Wang Y Q. Dawsonolagus antiquus, a primitive lagomorph from the Eocene Arshanto Formation, Nei Mongol, China. In: Beard K C, Luo Z X, eds. Mammalian Paleontology on a Global Stage: Papers in honor of Mary R. Dawson. Bull Carneg Mus Nat Hist, 2007, 39: 97-110

35 Bai B, Wang Y Q, Meng J. New craniodental materials of Litolophus gobiensis (Perissodactyla, Eomoropidae) from Inner Mongolia, China, and phylogenetic analyses of Eocene chalicotheres. Am Mus Novitat, 2010, 3688: 1—27, doi: 10.1206/678.1

36 Bai B. New materials of Eocene Dinocerata (Mammalia) from the Erlian Basin, Nei Mongol (Inner Mongolia). Vert PalAsiat, 2006, 44: $250-261$

37 Ni X J, Beard K C, Meng J, et al. Discovery of the first early Cenozoic euprimate (Mammalia) from Inner Mongolia. Am Mus Novitat, 2007, (3571): 1-11

38 Wang Y Q, Meng J, Ni X J, et al. A new Early Eocene arctostylopid (Arctostylopida, Mammalia) from the Erlian Basin, Nei Mongol (Inner Mongolia), China. J Vert Paleont, 2008, 28: 553-558 
39 Wang Y Q, Meng J, Beard K C, et al. Early Eocene perissodactyls from the upper Nomogen Formation of the Erlian Basin, Inner Mongolia, China. J Vert Paleont, 2008, 28: 175A

40 Szalay F S, Gould S J. Asiatic mesonychidae (mammalia, condylarthra). Bull Amer Mus Nat Hist, 1966, 132: 127-174

41 Granger W. A giant oxyaenid from the Upper Eocene of Mongolia. Am Mus Novitat, 1938, (969): 1-5

42 Granger W, Gregory W K. A revision of the Mongolian titanotheres. Bull Amer Mus Nat Hist, 1943, 80: 349-389

43 Radinsky L B. Early Tertiary tapiroidea of Asia. Bull Amer Mus Nat Hist, 1965, 129: 181-264

44 Meng J, Ni X J, Li C K, et al. New material of Alagomyidae (Mammalia, Glires) from the Late Paleocene Subeng locality, Inner Mongolia. Am Mus Novitat, 2007, (3579): 1-29

45 Missiaen P, Smith T. A new Paleocene nyctitheriid insectivore from Inner Mongolia (China) and the origin of Asian nyctitheriids. Acta Palaeont Pol, 2005, 50: 513-522

46 Smith T, Van Itterbeeck J, Missiaen P. Oldest Plesiadapiform (Mammalia, Proprimates) from Asia and its palaeobiogeographical implications for faunal interchange with North America. C R Palevol, 2004, 3: 43-52

47 Meng J, Wyss A R, Hu Y M, et al. Glires (mammalia) from the Late Paleocene Bayan Ulan locality of Inner Mongolia. Am Mus Novitat, 2005, (3473): 1-25

48 Wang Y Q, Tong Y S, Li Q. The Chinese continental Paleocene-Eocene boundary and its correlation. Acta Geol Sin, 2010, in press

49 Ting S Y, Bowen G J, Koch P L, et al. Biostratigraphic, chemostratigraphic, and magnetostratigraphic study across the Paleocene-Eocene boundary in the Hengyang Basin, Hunan, China. In: Wing S L, Gingerich P D, Schmitz B, et al, eds. Causes and Consequences of Globally Warm Climates in the Early Paleogene. Geol Soc Am Spec Paper, 2003, 369: 521—535

50 Wood H E, Chaney R W, Clark J, et al. Nomenclature and correlation of the North American continental Tertiary. Geol Soc Am Bull, 1941, 52: $1-48$

51 Woodburne M O. Definition and characterization in mammalian chronostratigraphy. J Paleont, 1977, 51: 220-234

52 Woodburne M O. Principles and Procedures. In: Woodburne M O, ed. Late Cretaceous and Cenozoic mammals of North America: Biostratigraphy and Geochronology. New York: Columbia University Press, 2004. 1-20

53 Lofgren D L, Lillegraven J A, Clemens W A, et al. Paleocene biochronology: The Puercan through Clarkforkian land mammal ages. In: Woodburne M O, ed. Late Cretaceous and Cenozoic Mammals of North America: Biostratigraphy and Geochronology. New York: Columbia University Press, 2004. 43-105

54 Prothero D R, Emry R J. The Chadronian, Orellan, and Whitneyan North American land mammal ages. In: Woodburne M O, ed. Late Cretaceous and Cenozoic Mammals of North America: Biostratigraphy and Geochronology. New York: Columbia University Press, 2004. $156-168$

55 Robinson P, Gunnell G F, Walsh S L, et al. Wasatchian through Duchesnean biochronology. In: Woodburne M O, ed. Late Cretaceous and Cenozoic Mammals of North America: Biostratigraphy and Geochronology. New York: Columbia University Press, 2004. 106-155

56 Tedford R H, Albright L B III, Barnosky A D, et al. Mammalian biochronology of the Arikareean through Hemphillian interval (Late Oligocene through Early Pliocene Epochs). In: Woodburne M O, ed. Late Cretaceous and Cenozoic Mammals of North America: Biostratigraphy and Geochronology. New York: Columbia University Press, 2004. 169-231

57 Woodburne M O. Global events and the North American mammalian biochronology. In: Woodburne M O, ed. Late Cretaceous and Cenozoic Mammals of North America: Biostratigraphy and Geochronology. New York: Columbia University Press, 2004. 315-344

58 Ting S Y. Paleocene and early Eocene land mammal ages of Asia. In: Beard K C, Dawson M R, eds. Dawn of the Age of Mammals in Asia. Bull Carneg Mus Nat Hist, 1998, 34: 127-147

59 Tong Y S, Zheng S H, Qiu Z D. Cenozoic mammal ages of China. Vert PalAsiat, 1995, 33: 290-314

60 Szalay F S, McKenna M C. Beginning of the age of mammals in Asia: The late Paleocene Gashato fauna, Mongolia. Bull Amer Mus Nat Hist, 1971, 144: 269-318

61 Dashzeveg D. Holarctic correlation of non-marine Palaeocene-Eocene boundary using mammals. J Geol Soc, London, 1988, 145: 473-478

62 Gingerich P D. Early Eocene Teilhardina brandti: Oldest omomyid primate from North America. Contrib Mus Paleont Univ Mich, 1993, 28: $321-326$

63 薛祥煦, 岳乐平, 张云翔. 陕西山阳盆地红色地层磁性、生物和岩石地层界线的对比. 中国科学 B 辑, 1994, 24: 413-417

64 赵资奎, 叶捷, 李华梅, 等. 广东省南雄盆地白严系-第三系交界恐龙绝灭问题. 古脊椎动物学报, 1991, 29: 1-20

65 Clyde W C, Ting S Y, Snell K E, et al. New paleomagnetic and stable-isotope results from the Nanxiong Basin, China: Implications for the K/T boundary and the timing of Paleocene mammalian turnover. J Geol, 2010, 118: 131-143

66 Clyde W C, Tong Y S, Snell K E, et al. An integrated stratigraphic record from the Paleocene of the Chijiang Basin, Jiangxi Province (China): Implications for mammalian turnover and Asian block rotations. Earth Planet Sci Lett, 2008, 269: 553-563

67 Zachos J C, Pagani M, Sloan L C, et al. Trends, rhythms, and aberrations in global climate 65 Ma to present. Science, 2001, 292: 686-693

68 Sluijs A, Brinkhuis H, Schouten S, et al. Environmental precursors to rapid light carbon injection at the Palaeocene/Eocene boundary. Nature, 2007, 450: 1218-1221 